\title{
A rational equation for predicting search times in simple inspection tasks.
}

\section{I. HOWARTH and J. R. BLOOMFIELD, University of Nottingham, Nottingham, England}

Often, in inspection tasks, the Ofaces the problem of detecting abnormal target elements in an array of nomal ones. From the known characteristics of the eye and from a simple theory of visual search, we have developed an equation relating the extent to which the target element deviates from normal to the time necessary to search for it. The equation fits data from a number of simple search experiments by us and by others. We think it should be of use in designing inspection systems. The equation also suggests how visual-search theory may be tested experimentally.

\section{DERIVATION OF THE EQUATION}

If an $O$ is searching visually within a two-dimensional area, $\mathbf{A}$, his mean search time, $\bar{t}$, should be given by the equation

$$
\bar{t}=\frac{t_{s} \cdot A}{a},
$$

providing he searches by making repeated fixations of duration, $t_{s}$, during which he can detect targets within an area, a, around the line of sight, and provided his fixations are randomly distributed over $A$. This equation gives the mean value of an exponential distribution.

If $t_{s}$, the fixation time, is unaffected by

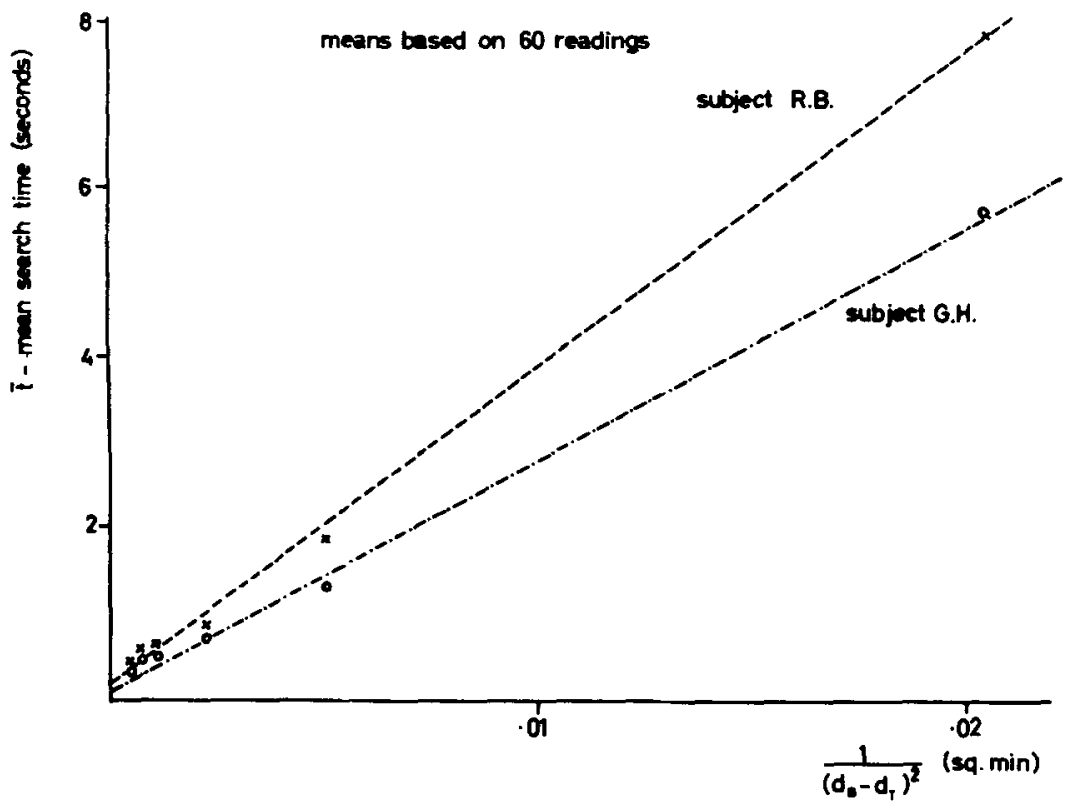

Assuming that the area covered by $a$ is circular,

$$
\mathrm{a}=\pi \cdot \theta^{2} .
$$

Therefore,

$$
\mathrm{a}=\frac{\pi\left(d_{B}-d_{T}\right)^{2}}{m^{2}} .
$$
and $a$, the area in which it can be detected in a single fixation, then we will have an equation relating mean search time to target characteristics.

The distance away from the line of sight at which objects of various sizes can be detected is well known for conventional acuity targets. Weymouth (1958) pointed out that there is a simple linear relationship between the size of the target and $\theta$ the distance from the line of sight at which the target can be detected, for values of $\theta$ up to 20 deg of eccentricity. In an inspection task involving discs of varying size, the problem is effectively to detect the difference in diameters of the normal or background elements and the abnormal or target elements, $d_{B}-d_{T}$. M. L. Matthews, in this department, confirmed that the threshold value of this difference is linearly related to the distance of targets from the foveal fixation point.

Thus,

$$
\left|\mathrm{d}_{\mathbf{B}}-\mathrm{d}_{\mathbf{T}}\right|=\mathrm{m} \cdot \theta,
$$

where $m$ is the gradient of the straight line obtained by plotting $\left|\mathrm{d}_{\mathrm{B}}-\mathrm{d}_{\mathrm{T}}\right|$ against $\theta$, and is, therefore, constant for constant experimental conditions. Therefore,

$$
\theta=\frac{\left|\mathrm{d}_{\mathbf{B}}-\mathrm{d}_{\mathbf{T}}\right|}{\mathrm{m}} .
$$

Substituting Eq. 3 in Eq. 1 gives

$$
\bar{t}=\frac{t_{s} \cdot A \cdot m^{2}}{\pi\left(d_{B}-d_{T}\right)^{2}} .
$$

This equation relates the size of the difference between the diameters of target and background stimuli to the mean time needed to find the target, and, since $t_{s}, A$, and $m$ should all be constant, it can be simplified to

$$
\bar{t} \propto \frac{1}{\left(d_{B}-d_{T}\right)^{2}} .
$$

In a real inspection task, the time one should allow for search should be some multiple of the mean search time, depending on the proportion of abnormal elements that can be tolerated, and on the proportion that are expected.

We have assumed that search is effectively random-the work of Krendel \& Wodinsky (1960) provides considerable support for this assumption. We have ignored the fact that the probability of detection does not fall sharply from $100 \%$ to $0 \%$ at a certain distance, $\theta$, from the fovea. This is justified, since a fuller and more realistic treatment makes almost no difference to the predictions and none at all to the simpler form of our search equation, which we have tested. We have also assumed that mean fixation time, $t_{s}$, is independent of the search task. This is almost certainly not the case and deserves rigorous experimental investigation. It is worth noting that every term in our search equation (Eq. 4) can be evaluated independently by experiment. TESTING THE EQUATION

We have tested the equation in two situations with very different characteristics. In the first, the 0 had to detect a smaller disc in an array of 100 normal ones. Both regular and irregular arrangements of the discs have been used. Figure 1 shows how well the data from two

Fig. 1. The relationship between $\overline{\mathbf{t}}$ and $1 /\left[\left(d_{s}-d_{t}\right)^{2}\right]$ for two Ss searching a regularly arranged display of 100 dises; the target discs were all, to varying degrees, smaller in size. 


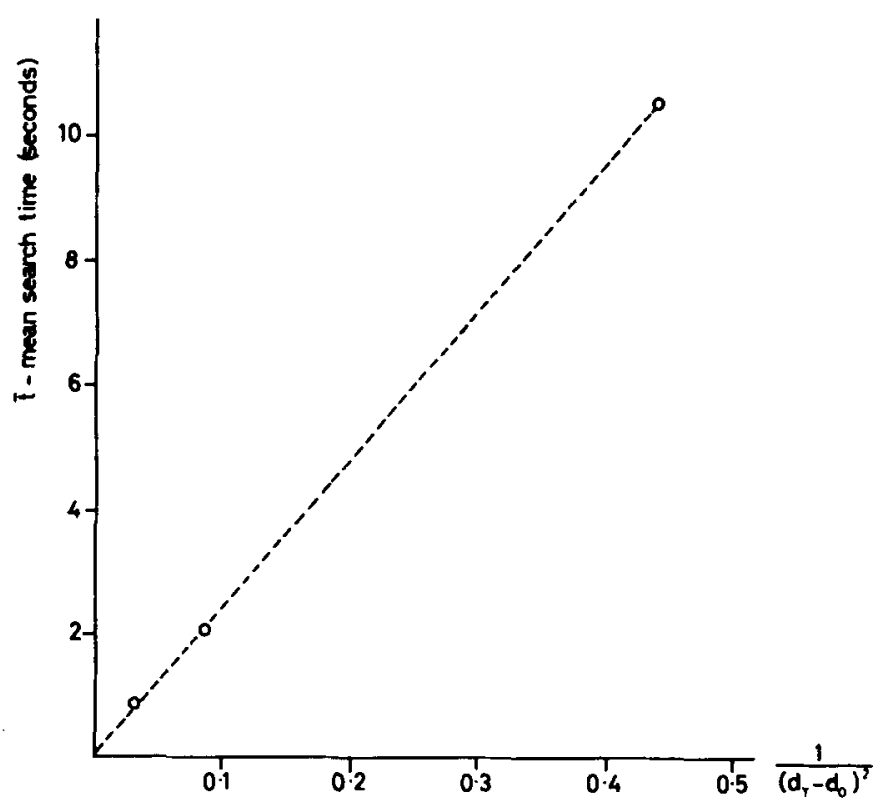

of our Ss searching a regular display fits Eq. 5 . We have very extensive data from this experimental situation, all of which is equally well described by the equation.

In the second situation, the $O$ had to detect a small square of very low contrast, randomly positioned on a plain CRT display. By reworking data from J. H. Taylor, reported by Linge (1961), on lowcontrast targets, we found that, for this situation, Eq. 2 should have the form

$$
\theta=\frac{\left(\mathrm{d}_{\mathrm{T}}-\mathrm{d}_{\mathrm{o}}\right)}{\mathrm{m}},
$$

where $d_{0}$ is the threshold diameter of a target on the line of sight, and $d_{T}$ is the diameter of the target disc. With this value for $\theta$, Eqs. 4 and 5 become

$$
\overrightarrow{\mathrm{t}}=\frac{\mathrm{t}_{\mathrm{s}} \cdot \mathrm{A} \cdot \mathrm{m}^{2}}{\pi\left(\mathrm{d}_{\mathrm{T}}-\mathrm{d}_{\mathrm{o}}\right)^{2}}
$$

and

$$
\bar{t} \propto \frac{1}{\left(d_{T}-d_{o}\right)^{2}} .
$$
data from this experiment. this to be true.
Fig. 2. The relationship between $\bar{t}$ and $1 /\left[\left(d_{t}-d_{0}\right)^{2}\right]$, where $d_{0}=2.5$, for search for varying sizes of disc in a plain background.

the effects are quite complicated. Second, an increase in density may act as "visual noise," which, as Mackworth (1965) has shown, produces "tunnel vision." This reduction in peripheral acuity should lead to an increase in search times. Smith (1962) showed there is a considerable increase in mean search time, with an increase in the density of nontarget stimuli. The second of the two effects appears to have much the greater influence, and we believe it can be included fairly simply in our equation.

\section{REFERENCES}

ERICKSON, R. A. Relation between visual search time and peripheral visual acuity. Human Factors, 1964, 6, 165-177.

JOHNSTON, D. M. Search performance as a function of peripheral acuity. Human Factors, $1965,7,527-535$.

Figure 2 shows how well Eq. 8 describes the

Other predictions from Eq. 4 can be confirmed from existing data. One would expect viewing distance to have no effect, and this seems to be the case (Parkes, 1967). Search time should be proportional to search area, and Krendel \& Wodinsky (1960) found it was. Os with good peripheral vision should detect targets more quickly than those with poor peripheral vision. Erickson (1964) and Johnston (1965) have shown

Varying the density of normal, nontarget elements might be expected to produce two opposing effects. First, an increase in density would increase the proximity of target and nontarget stimuli. This might be expected to make detection quicker. M. L. Matthews has shown, in our laboratories, that this is not necessarily the case and that
KRENDEL, E. S., \& WODINSKY, J. Visual search in an unstructured visual field. Journal of the Optical Society of America, 1960, 50,562-568.

LINGE, A. Visual detection from aircraft. General Dynamics/Convair Engineering Research Report. ASTIA 270630, 1961.

MACKWORTH, N. H. Visual noise causes tunnel vision. Psychonomic Science, 1965, 3, 67-68.

PARKES, K. R. Visual and televisual detection studies: II. The effect of viewing distance on target detection performance. Loughborough University of Technology Research Report, 1967.

SMITH, S. W. Problems in the design of sensor output displays. In M. A. Whitcomb (Ed.), Visual problems of the Armed Forces. Washington, D.C.: National Academy of Sciences-National Research Committee, 1962. Pp. 146-157. minimal angle of resolution. American Journal of Opthalmology, 1958,41, 102-113.

$$
\text { NOTE }
$$

1. We thank Dr. E.B. Davies and Mr. F.I. Reynolds of the Royal Aircraft Establishment, Farnborough, for many helpful discussions of the problems involved in this work. We also thank Mr. . F. Kemp, who has permitted us to use some of his data for Fig. 2.
WEYMOUTH, F. W. Visual sensory units and the 